\title{
Equivalent Circuit Parameters Estimation for PEM Fuel Cell Using RBF Neural Network and Enhanced Particle Swarm Optimization
}

\author{
Wen-Yeau Chang \\ Department of Electrical Engineering, St. John's University, 499, Sec. 4, Tam King Road, Tamsui District, New Taipei City 25135, Taiwan \\ Correspondence should be addressed to Wen-Yeau Chang; changwy@mail.sju.edu.tw
}

Received 13 December 2012; Revised 30 January 2013; Accepted 13 February 2013

Academic Editor: Quang Phuc Ha

Copyright (C) 2013 Wen-Yeau Chang. This is an open access article distributed under the Creative Commons Attribution License, which permits unrestricted use, distribution, and reproduction in any medium, provided the original work is properly cited.

\begin{abstract}
This paper proposes an equivalent circuit parameters measurement and estimation method for proton exchange membrane fuel cell (PEMFC). The parameters measurement method is based on current loading technique; in current loading test a no load PEMFC is suddenly turned on to obtain the waveform of the transient terminal voltage. After the equivalent circuit parameters were measured, a hybrid method that combines a radial basis function (RBF) neural network and enhanced particle swarm optimization (EPSO) algorithm is further employed for the equivalent circuit parameters estimation. The RBF neural network is adopted such that the estimation problem can be effectively processed when the considered data have different features and ranges. In the hybrid method, EPSO algorithm is used to tune the connection weights, the centers, and the widths of RBF neural network. Together with the current loading technique, the proposed hybrid estimation method can effectively estimate the equivalent circuit parameters of PEMFC. To verify the proposed approach, experiments were conducted to demonstrate the equivalent circuit parameters estimation of PEMFC. A practical PEMFC stack was purposely created to produce the common current loading activities of PEMFC for the experiments. The practical results of the proposed method were studied in accordance with the conditions for different loading conditions.
\end{abstract}

\section{Introduction}

Soaring crude oil price and exacerbating influence of emission on environment are leading to the exploitation of renewable energy technologies. The fuel cell is one of the most appealing energy conversion devices in renewable energy systems because of its high potential commercial applications [1]. The fuel cell system is an electrochemical system converting chemical energy to electricity and thermal energy. The advantages of fuel cells can be summarized as follows: simplicity, minimal environmental impact, efficiency, and silence. Promising applications for fuel cells include portable power for electronics appliances, electric vehicles, building cogeneration systems, hybrid energy system, and distributed power generation systems for utilities.

There are several kinds of fuel cells currently being investigated for use in industry: PEMFC [2], solid oxide fuel cell, direct methanol fuel cell, phosphoric acid fuel cell, molten carbonate fuel cell, and aqueous alkaline fuel cell. In these kinds of fuel cells, PEMFC is being rapidly developed as the primary power source in electric vehicles and distributed power generation systems, because of its firm and simple structure, high energy density, and low working temperature.

Because the interest in PEMFC grows, the urge for accurate simulation models grows as well. Since PEMFC system is a multi-input, multioutput electrochemical system, the exact electrochemical processes of PEMFC can be described by mathematical models. Mathematical models can improve the design of the flow fields, membranes, and catalysts, as well as it can determine the optimal operating conditions such as humidity, temperature, and fuel flow rates [3]. However, such mathematical models are not well suited for describing the electrical behavior of the fuel cell or how it will interact with power electronics circuits such as inverters.

Therefore, equivalent circuit models of PEMFC are widely used to describe its macroscopic behavior, which has real 
importance in the exploitation phase of PEMFC. If parameters estimation is used for monitoring purposes during exploitation, then useful information can be obtained about behavior of PEMFC. Equivalent circuit model of PEMFC is necessary to simulate the behavior of more complex power conversion systems such as electric vehicles and distributed power generation systems, in which PEMFC is integrated as a source of power [4].

Recently, RBF neural network methods have received a great deal of attention and were proposed as powerful computational tools to solve the parameter estimation problem. RBF neural network is applied to estimate the equivalent circuit parameters of PEMFC in this paper. The RBF neural network provides a universal approximation, and basis functions in the hidden layer of RBF neural network are utilized. The RBF neural network can determine implicit nonlinear relationships between input variables by learning from training data.

In this paper, the EPSO algorithm is applied to the RBF neural network in the training phase, to obtain a set of weights, centers, and widths that will minimize the error function in competitive time. Weights, centers, and widths are progressively updated until the convergence criterion is satisfied. The objective function to be minimized by the EPSO algorithm is the estimated error function. The most prominent merit of EPSO algorithm is its fast convergence speed. In addition, EPSO algorithm can be computationally inexpensive and easily implemented and does not require gradient information of an objective function but only its values.

This paper deals with equivalent circuit parameters estimation of PEMFC and is divided in to nine sections. After a brief introduction, Section 2 discusses the equivalent circuit models of PEMFC. Section 3 is entirely dedicated to the parameters measurement method. Section 4 introduces the principle of RBF neural network. Section 5 describes the principle of EPSO algorithm. Section 6 describes EPSO based RBF neural network training procedure. Section 7 discusses the RBF neural network based parameters estimation method. Experimental results are described in Section 8. The conclusions of the paper are summarized in Section 9.

\section{Equivalent Circuit Models of PEMFC}

Equivalent circuit models of PEMFC would aid in the design and control of the interfacing electronics and designing and analyzing reliability tests. The equivalent circuit models of PEMFC can be generally categorized into two types: dynamic models and passive models [5]. The dynamic equivalent circuit model represents the operating behavior of PEMFC under the output electric power state. The dynamic models must include a voltage source and the inherent losses of PEMFC. These kinds of models can be used to optimize the performance, transient response, and efficiency of power conversion systems using PEMFC as the power source of systems. The passive equivalent circuit model represents the operating behavior of PEMFC in the stand-by state. The passive models can be employed to determine the potential

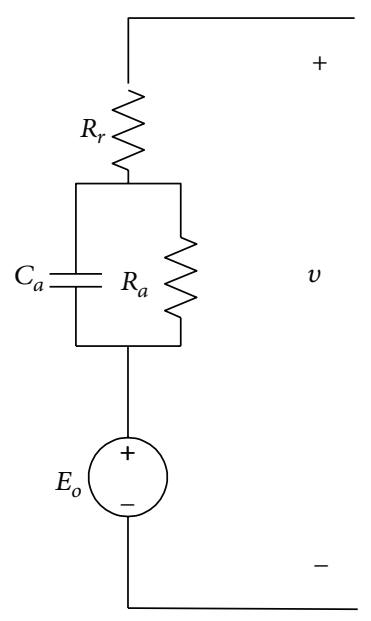

FIGURE 1: The simplified equivalent circuit mode of PEMFC.

performance and degradation of the PEMFC while it is in a stand-by mode [3].

This paper examines the dynamic equivalent circuit models of PEMFC. In this paper we choose the simplified equivalent circuit shown in Figure 1 to model the PEMFC [6]. In the simplified equivalent circuit model, the dynamic behavior of a PEMFC can be represented by a simple firstorder equivalent circuit. Considering the main losses of PEMFC, the activation and ohmic losses, an equivalent circuit model of PEMFC can be derived.

The components of equivalent circuit are described as follows. The ohmic losses are represented through the resistor $\left(R_{r}\right)$ which expresses the internal resistance of the PEMFC, such as the resistance of electrodes, the resistance of conductive plates, and the resistance of proton which is transferring through the membrane. The activation losses are represented through the parallel connection of a resistor $\left(R_{a}\right)$ with a capacitor $\left(C_{a}\right)$ that models the double layer of charge at the interfaces between the membrane and the electrodes. The dc voltage source $\left(E_{o}\right)$ is the theoretical open circuit voltage of the PEMFC. In this paper the current loading technique is used for obtaining PEMFC parameters mentioned above. The current loading technique is introduced in the next section.

\section{Current Loading Technique for Parameters Measurement}

The current loading technique is easy to perform with a single PEMFC and small PEMFC stack, but even for a larger PEMFC stack, switching higher current is not problematic. The principle of the current loading technique is described as follow [5].

The test circuit needed to perform the current loading technique is shown in Figure 2. During the current loading test, a no load PEMFC is suddenly turned on and the terminal voltage of PEMFC will gradually reach the steady state. The main properties that can be obtained using current loading technique are the ohmic resistance obtained from the instantaneous change in terminal voltage and activation loss 


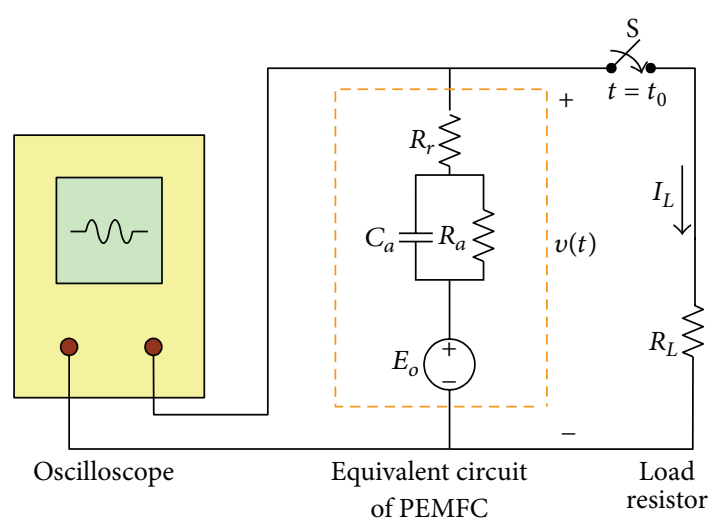

FIGURE 2: The test circuit for performing current loading technique.

calculated from the gradual change in terminal voltage to a steady state value. The waveform of the terminal voltage and load current will change as shown in Figures 3(a) and 3(b).

At time $t=t_{0}$ the switch $\mathrm{S}$ is closed. Figure 3(a) shows the terminal voltage corresponding to time $t<t_{0}$ can be expressed as

$$
v\left(t<t_{0}\right)=E_{0} .
$$

The terminal voltage corresponding to time $t>t_{0}$ can be obtained from the following equation:

$v(t)=\frac{R_{L}}{R_{L}+R_{a}+R_{r}}\left(1-e^{-\left(t-t_{0}\right) / \tau}\right) E_{0}+\frac{R_{L}}{R_{L}+R_{r}} e^{-\left(t-t_{0}\right) / \tau} E_{0}$,

where $\tau$ is the time constant of the test circuit, $\tau=C_{a}\left(R_{a} R_{L}+\right.$ $\left.R_{a} R_{r}\right) /\left(R_{a}+R_{L}+R_{r}\right)$.

The terminal voltage corresponding to time $t=t_{0}^{+}$, where $t_{0}^{+}$is the time immediately after $t_{o}$, is given as follows:

$$
v\left(t=t_{0}^{+}\right)=\frac{R_{L}}{R_{L}+R_{r}} E_{0} .
$$

The steady state terminal voltage after the switch is closed can be calculated by

$$
v_{\mathrm{ss}}=\frac{R_{L}}{R_{L}+R_{r}+R_{a}} E_{0} .
$$

Comparing (3) with (1) gives

$$
\frac{v\left(t=t_{0}^{+}\right)}{v\left(t<t_{0}\right)}=\frac{R_{L}}{R_{L}+R_{r}} .
$$

Solving (5) for the ohmic losses resistor $R_{r}$ gives the following expression:

$$
R_{r}=\frac{v\left(t<t_{0}\right)}{v\left(t=t_{0}^{+}\right)} R_{L}-R_{L} .
$$

Comparing (4) with (1) yields

$$
\frac{v_{s s}}{v\left(t<t_{0}\right)}=\frac{R_{L}}{R_{L}+R_{r}+R_{a}} .
$$

Solving (7), the activation losses resistor $R_{a}$ can be derived from the following expression:

$$
R_{a}=\frac{v\left(t<t_{0}\right)}{v_{\mathrm{ss}}} R_{L}-R_{L}-R_{r} .
$$

Using (2), the terminal voltage corresponding to time $t=$ $t_{0}+\tau$ can be calculated by

$$
\begin{aligned}
v\left(t=t_{0}+\tau\right)= & \frac{R_{L}}{R_{L}+R_{a}+R_{r}}\left(1-e^{-1}\right) E_{0}+\frac{R_{L}}{R_{L}+R_{r}} e^{-1} E_{0} \\
= & 0.632 \times \frac{R_{L}}{R_{L}+R_{a}+R_{r}} E_{0}+0.368 \\
& \times \frac{R_{L}}{R_{L}+R_{r}} E_{0} .
\end{aligned}
$$

Once the values of $E_{0}, R_{a}, R_{L}$, and $R_{r}$ are known, the value of $v\left(t_{0}+\tau\right)$ can be calculated from (9). Then the four terminal voltage values $v\left(t=t_{0}^{+}\right), v\left(t<t_{0}\right), v\left(t=t_{0}+\tau\right)$, and $v_{\text {ss }}$ and time interval $\tau$ can be easily read from the waveform of the terminal voltage of PEMFC, as shown in Figure 3(a).

Once the value of the time constant $\tau$ is known, the activation losses capacitor $C_{a}$ can be derived from the time constant

$$
C_{a}=\frac{R_{a}+R_{r}+R_{L}}{R_{a}\left(R_{r}+R_{L}\right)} \tau .
$$

After the current loading test, a hybrid parameters estimation method that combines an RBF neural network and EPSO algorithm is further employed for the equivalent circuit parameters estimation. The principles of RBF neural network and EPSO algorithm are described in the following sections.

\section{Principle of RBF Neural Network}

TheRBF neural network is a useful methodology for systems with incomplete information. It can be used to analyze the relationships between one major (reference) sequence and the other comparative ones in a given set [7]. The RBF neural network is a forward networks model with good performance and global approximation [8]. In order to reduce the local minima problem of RBF neural network, the EPSO algorithm is used to train the RBF neural network. In this section, the principle of RBF neural network is described.

It is a multi-input, multioutput system consisting of an input layer, a hidden layer, and an output layer. During data processing, the hidden layer performs nonlinear transforms for the feature extraction and the output layer gives a linear combination of output weights [9]. The structure is shown in Figure 4.

The network actually performs a nonlinear mapping from the input space $R^{d}$ to the output space $R^{n}$. The mapping relationship between input vector and output vector of $R B F$ neural networks is based on the following function:

$$
\text { RBF Neural Network : }\left\{\begin{array}{l}
R^{d} \longrightarrow R^{n} \\
\bar{y} \longrightarrow \bar{o},
\end{array}\right.
$$




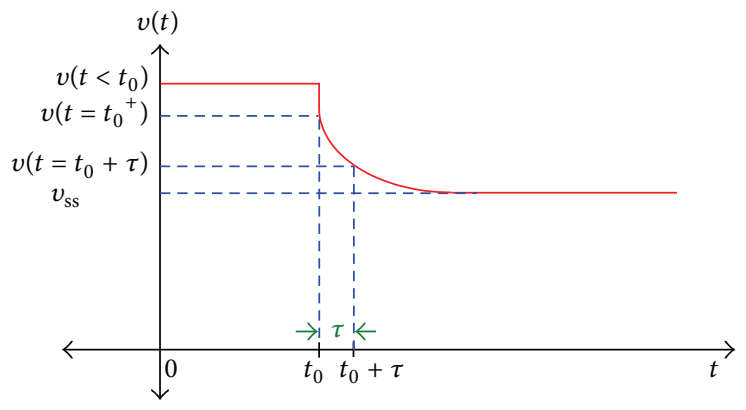

(a) Voltage

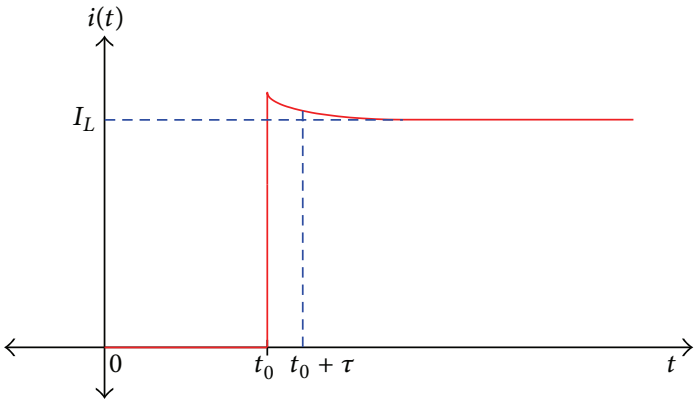

(b) Current

FIgURE 3: The waveform of voltage and current of a PEMFC while current loading.

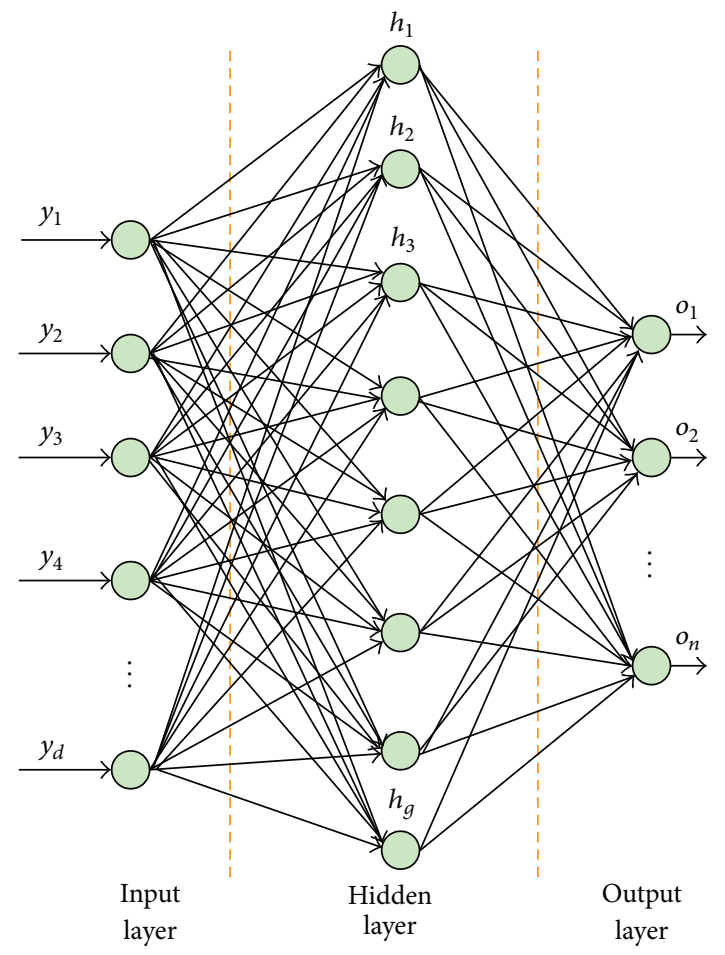

FIgURE 4: Architecture of the RBF neural network.

where input vector $\bar{y}_{i}=\left\{y_{i}\right.$, for $\left.i=1,2, \ldots, d\right\}$ and output vector $\bar{o}_{i}=\left\{o_{i}\right.$, for $\left.i=1,2, \ldots, n\right\}$.

Each hidden neuron computes a Gaussian function in the following equation:

$$
h_{j}(\bar{y})=\exp \left[\frac{-\left(\bar{y}-\mu_{j}\right)^{2}}{2 \sigma_{j}^{2}}\right], \quad \text { for } j=1,2, \ldots, g \text {, }
$$

where $\mu_{j}$ and $\sigma_{j}$ are the center and the width of the Gaussian potential function of the $j$ th neuron in the hidden layer, respectively.
Each output neuron of the RBF neural network computes a linear function in the following form:

$$
o_{k}=\sum_{j=1}^{g} w_{k j} h_{j}(\bar{y})+\theta_{k}, \quad \text { for } k=1,2, \ldots, n,
$$

where $o_{k}$ is output of the $k$ th node in the output layer, $w_{k j}$ is weight between $j$ th node in the hidden layer and $k$ th node in the output layer, $h_{j}(\bar{y})$ is output from the $j$ th node in the hidden layer, and $\theta_{k}$ is bias of the $k$ th node in the output layer.

\section{Principle of EPSO Algorithm}

The particle swarm optimization (PSO) algorithm is a heuristic approach developed for dealing with the optimization of continuous and discontinuous function decision making [10]. The PSO performs global search for objective function space to achieve a preliminary solution specifying a local potential space [11]. The PSO simulates the behavior of a swarm as a simplified social system. In a PSO system, each particle adjusts its position in light of its own experience and the experiences of swarm [12]. The social sharing of information among the particles of a population may provide an evolutionary advantage [13].

In standard PSO algorithm, particles are manipulated according to (14) and (15) where each particle tries to adjust its velocity according to the best previous position that is stored in its memory called personal best (denoted by pbest) and according to the best previous position attained by any particle in swarm called global best (denoted by gbest) trying to search for a better position [14]. The movement equation of $i$ th particle is as follows:

$$
\begin{aligned}
v_{i}(t+1)= & \omega v_{i}(t)+\eta_{1} \times r_{1}\left[\text { pbest }-x_{i}(t)\right]+\eta_{2} \\
& \times r_{2}\left[\text { gbest }-x_{i}(t)\right] \quad i=1,2,3, \ldots, N, \\
& x_{i}(t+1)=x_{i}(t)+v_{i}(t+1),
\end{aligned}
$$

where $v_{i}(t)$ and $v_{i}(t+1)$ are current and modified velocities for each iteration, respectively. $\eta_{1}$ and $\eta_{2}$ are positive numbers, used to control the particle's movement at each iteration. They represent cognitive and social components, respectively. $r_{1}$ and $r_{2}$ are uniform distribution numbers in the range $[0,1]$. 
$x_{i}(t)$ and $x_{i}(t+1)$ are the current and modified positions for each iteration, respectively. $N$ is the number of particles. $\omega$ denotes the inertial weight, which is set according to the following [10]:

$$
\omega=\omega_{\max }-\frac{\omega_{\max }-\omega_{\min }}{t_{\max }} \times t_{n},
$$

where $t_{\max }$ is the maximum number of iterations and $t_{n}$ is the current number of iterations. Equation (16) restricts the value $\omega$ to the range $\left[\omega_{\max }, \omega_{\min }\right]$.

Equation (14) reveals that the search procedure by PSO heavily depends on pbest and gbest. If the initial population of particles cannot effectively cover the whole region, the particles will converge usually on a local optimal solution not a global optimal solution. To avoid these limitations, in this paper a mutation mechanism of evolutionary computation is then incorporated into PSO algorithm called enhanced particle swarm optimization (EPSO) algorithm [12]. In general, the particles with poor individuals are selected for mutation from a subset of the swarm.

The next position of the $i$ th particle selected for mutation will be modified by

$$
x_{i}(t+1)=x_{i}(t)+N\left(0, \sigma_{i}^{2}\right) \quad i=1,2,3, \ldots, Z,
$$

where $N\left(0, \sigma_{i}^{2}\right)$ is a vector of Gaussian random variables with mean zero and standard deviation $\sigma_{i}, i=1,2, \ldots, Z$, in which $Z$ is the number of particles selected for mutation. Generally, $Z$ is set at $10 \%$ of population. In addition, $\sigma_{i}$ in (17) is set according to the following:

$$
\sigma_{i}=\lambda \| g \text { best }-x_{i}(t) \|+O,
$$

where $\|$ gbest $-x_{i}(t) \|$ denotes the Euclidean distance between $x_{i}(t)$ and gbest. $\lambda$ is the scaling factor and $O$ represents an offset.

As given in (17) and (18), the next position of the $i$ th particle selected for mutation is attained according to the distance between $x_{i}(t)$ and gbest. If the distance is relatively large, the next position of $i$ th selected particle will be searched over a wider range.

An EPSO algorithm is used to train the RBF neural network by tuning the position of RBF centers, the widths of RBFs, and the connection weights. The training procedure will be introduced in the following section.

\section{EPSO Based RBF Neural Network Training Procedure}

The EPSO based RBF neural network training procedure is described as follows [12].

Step 1 (initialization). Initialize the positions and velocities of a group of particles. Randomly generate the initial trial vectors $\left(x_{i}(0)=1,2, \ldots, N, N\right.$ is the number of particles), which indicate the possible solutions for the position of RBF centers, the widths of RBFs, and the weights between hidden and output layers. The element in vector $x_{i}(0)$ is randomly generated as follows:

$$
x_{i} \in U\left[x_{\min }, x_{\max }\right] \quad i=1,2, \ldots, N,
$$

where $U\left[x_{\min }, x_{\max }\right]$ designates the outcome of a uniformly distributed random variable ranging over the given lowerbounded values $x_{\min }$ and upper-bounded values $x_{\max }$.

Step 2 (evaluation of fitness value). Evaluate each particle's fitness value; for each trial vector $x_{i}(t)$, a fitness value should be assigned and evaluated. The criterion of mean squared error function defined below is adopted to stand for the fitness value of the RBF network

$$
J=\frac{1}{n} \sum_{k=1}^{n}\left(o_{k}-\widehat{o}_{k}\right)^{2},
$$

where $\widehat{o}_{k}$ is the computed output of the RBF network by using (13), $o_{k}$ is the corresponding actual output, and $n$ is the number of network output nodes.

Step 3 (selection and memorization). Each particle $x_{i}$ memorizes its own fitness value and chooses the minimum one that has been better so far as pbest ${ }_{i}$. Then we have pbest $=$ $\left[\right.$ pbest $_{1}$, pbest $_{2}, \ldots$, pbest $\left._{N}\right]$ in the population. Besides, each particle also memorizes the other particles' fitness values to know their experiences. The particle with the best fitness value among pbest is denoted by gbest. Note that in the first iteration, each particle $x_{i}$ is set directly to pbest ${ }_{i}$ and the particle with the best fitness value among pbest is set to gbest.

Step 4 (modification of the velocity and position). Using (14) and (15), the positions and velocities of all the particles are modified.

Step 5 (mutation). In Steps 3 to 4 , the particles are ranked in a descending order of their corresponding fitness value. The particles with bad fitness values are selected for mutation from swarm subset by using (17) and (18).

Step 6 (stopping rule). Repeat Steps 2 to 5 until the best fitness value (gbest) can satisfy the minimum requirement or the given count of total generations is reached. The solution with the lowest fitness value is chosen as the best parameter in the RBF network that shall further be applied to equivalent circuit parameters estimation.

\section{RBF Neural Network Based Parameters Estimation Method}

The proposed RBF neural network based equivalent circuit parameters estimation method has been successfully implemented for the PEMFC parameters estimation. The architecture of the proposed RBF neural network is shown in Figure 5. The architecture of RBF neural network used in this study contains an input layer, an output layer, and a hidden layer. The input layer has 2 neurons for $I_{L}$ and $v_{s s}$, the hidden layer has 7 neurons, and the output layer has 3 neurons for $R_{r}$, $R_{a}$, and $C_{a}$.

The proposed equivalent circuit parameters estimation method is described briefly in the following steps:

Step 1. Creating database of the equivalent circuit parameters of PEMFC by using current loading technique. 


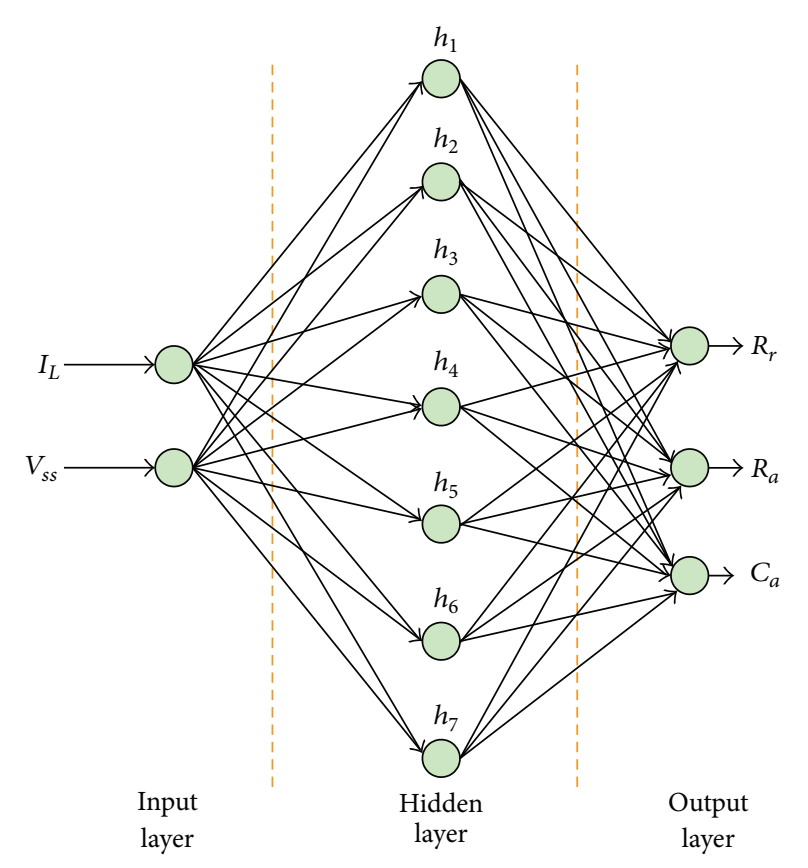

FIGURE 5: The architecture of the RBF neural network used in this paper.

Step 2. Normalize all of the equivalent circuit parameters data.

Step 3. Prepare the training set for RBF neural network.

Step 4. Using the EPSO algorithm to train the RBF neural network for equivalent circuit parameters estimation.

Step 5. Save the Gaussian functions centers and widthsand connection weights between the hidden and output layers of trained RBF neural network, as the EPSO based training procedure is finished.

Step 6. Use trained RBF neural network to estimate the equivalent circuit parameters of PEMFC.

To verify the proposed parameters measurement and estimation method, experiments were conducted to demonstrate its effectiveness. The experiment results are discussed in the following section.

\section{Experiment Results}

The experimental parameters measurement results were detected from a PEMFC test system, which was set up in our laboratory. The PEMFC test system is composed of an auxiliary system for cell condition regulation and a PEMFC stack. The block diagram and actual hardware structure of the PEMFC test system are shown in Figures 6 and 7. The PEMFC stack is a series of 12 membrane electrode assemblies comprising a Nafion 112 membrane with a thickness of $50 \mu \mathrm{m}$. The active area of the electrodes is $50 \mathrm{~cm}^{2}$. The system has a
TABLE 1: Estimation performance of testing data.

\begin{tabular}{lcc}
\hline Data base & Level of load current & Accuracy rate \\
\hline & Level 1 $(0 \sim 0.8 \mathrm{~A})$ & $94.2 \%$ \\
& Level $2(0.8 \sim 1.6 \mathrm{~A})$ & $95.1 \%$ \\
& Level 3 $(1.6 \sim 2.4 \mathrm{~A})$ & $93.7 \%$ \\
& Level $4(2.4 \sim 3.2 \mathrm{~A})$ & $95.5 \%$ \\
& Level 5 (3.2 4.0 A) & $95.8 \%$ \\
& Level 6 (4.0 4.8 A) & $96.2 \%$ \\
& Level 7 (4.8 5.6 A) & $96.8 \%$ \\
Testing data (120 patterns) & Level 8 (5.6 6.4 A) & $95.6 \%$ \\
& Level 9 (6.4 7.2 A) & $94.7 \%$ \\
& Level 10 (7.2 8.0 A) & $95.2 \%$ \\
& Level 11 (8.0 8.8 A) & $95.6 \%$ \\
& Level 12 (8.8 9.6 A) & $95.7 \%$ \\
Level 13 (9.6 10.4 A) & $96.2 \%$ \\
Level 14 (10.4 11.2 A) & $96.4 \%$ \\
Level 15 (11.2 12.0 A) & $95.4 \%$ \\
\hline
\end{tabular}

rated output power of $100 \mathrm{~W}$ at a voltage of $8 \mathrm{~V}$ and the opencircuit voltage is $11.4 \mathrm{~V}$.

The PEMFC test system has been conducted to operate at steady state conditions, with the following test parameters: (a) pressures of hydrogen and air, respectively, of 0.2 Psig and 1.13 Psig; (b) temperatures of hydrogen and air, respectively, of $64^{\circ} \mathrm{C}$ and $61^{\circ} \mathrm{C}$; (c) flow rates of hydrogen and air, respectively, of $2.5 \mathrm{slpm}$ and $11.0 \mathrm{slpm}$; (d) relative humidity of hydrogen and air, respectively, of $100 \%$.

Parameters measurement test using current loading technique has been performed for 15 different levels of load current (set from $0.8 \mathrm{~A}$ to $12 \mathrm{~A}$ ), and each load current level has 20 different types of load current. These different types of load current have 300 load current events data.

A typical current loading testing result is demonstrated below [15]. The voltage variation of the PEMFC during and after the loading is shown in Figure 8, which corresponds to the operating point obtained for $R_{L}=10.4 \Omega$. In Figure 8, Channel 1 denotes the waveform of terminal voltage ( $2 \mathrm{~V} / \mathrm{div})$, Channel 2 indicates the waveform of load current ( $1 \mathrm{~A} / \mathrm{div}$, the transfer rate of current probe is $1 \mathrm{~A} / 100 \mathrm{mV}$ ), and time base is $500 \mathrm{~ms} / \mathrm{div}$. As shown in Figure 8, $v(t=$ $\left.t_{0}^{+}\right)=10.800 \mathrm{~V}, v\left(t<t_{0}\right)=11.302 \mathrm{~V}, v_{\mathrm{ss}}=9.234 \mathrm{~V}$, and $I_{L}=0.8879 \mathrm{~A}$. Using (6), (8), and (10), the parameters of the equivalent circuit were obtained as follows: $R_{r}=$ $0.4833 \Omega, R_{a}=1.8454 \Omega$, and $C_{a}=0.06654 \mathrm{~F}$. The simulated results of the obtained equivalent circuit model using ISPICE simulation software are shown in Figure 9. Comparing Figure 8 with Figure 9, there is a good agreement between the simulated and experimental results by using current loading technique.

After current loading tests for parameters measurement, associated with their load current events, there are a total of 300 sample data for different load current events. Each load current level contains 20 load current vectors of sample data. For each load current level the training data consist of 12 load current vectors, which are randomly chosen from the 


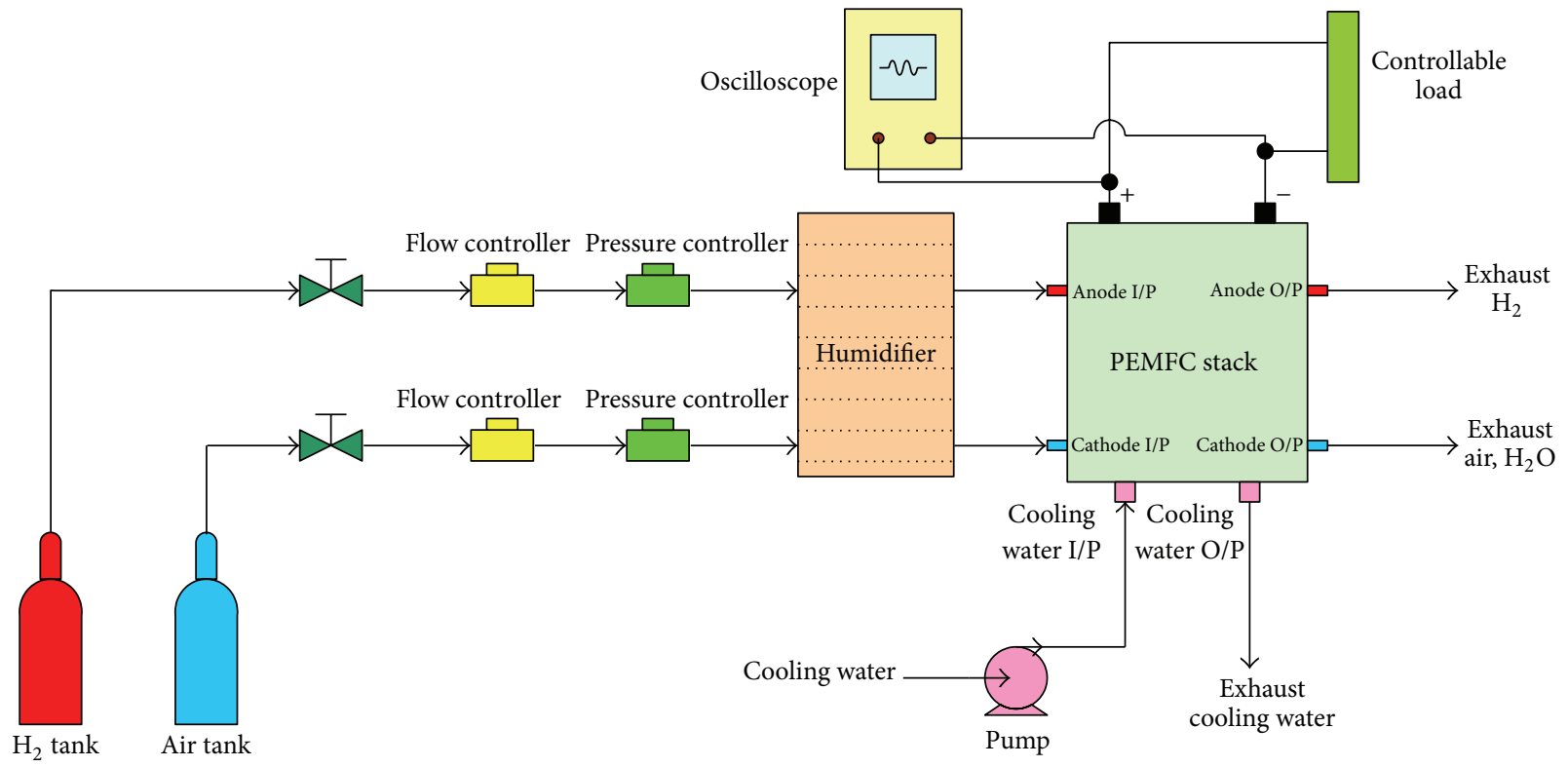

FIGURE 6: The block diagram of PEMFC test system.

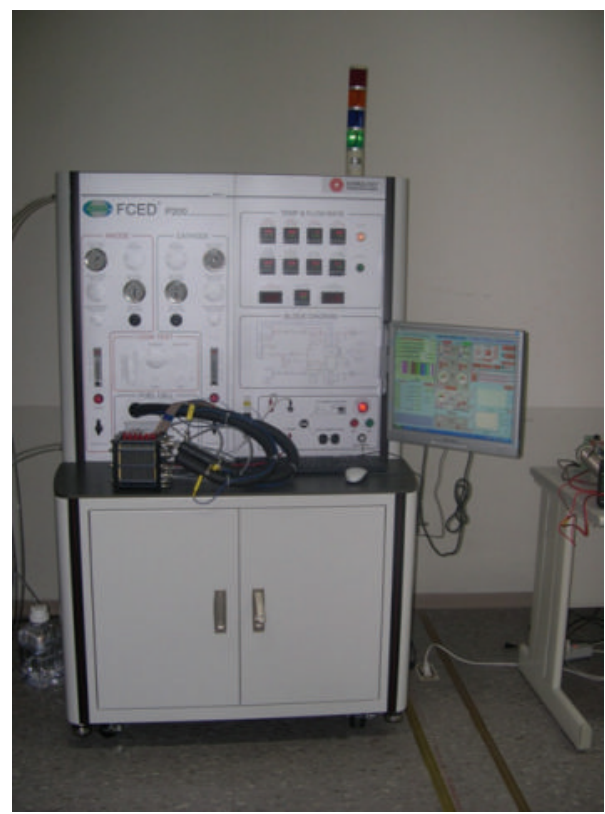

FIGURe 7: Actual hardware structure of the PEMFC test system.

20 vectors of sample data. The rest of 8 load current vectors were used as the testing data. After setting up the sample sets, the EPSO based training procedure of RBF neural network is started. The training data consist of 180 load current vectors, and the rest of 120 load current vectors were used as the testing data.

To verify the training results of RBF neural network, the training data were applied to the trained RBF neural network again. The data show that the proposed method has $100 \%$ accuracy rate for the 180 training vectors.

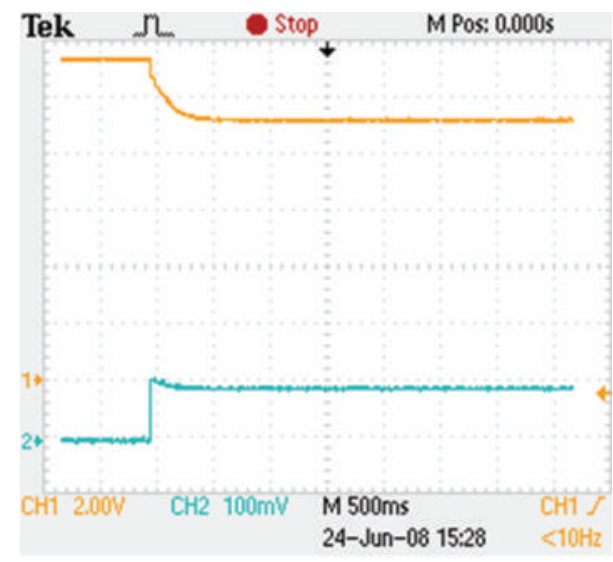

FIgURE 8: The experimental waveform of voltage and current for current loading testing.

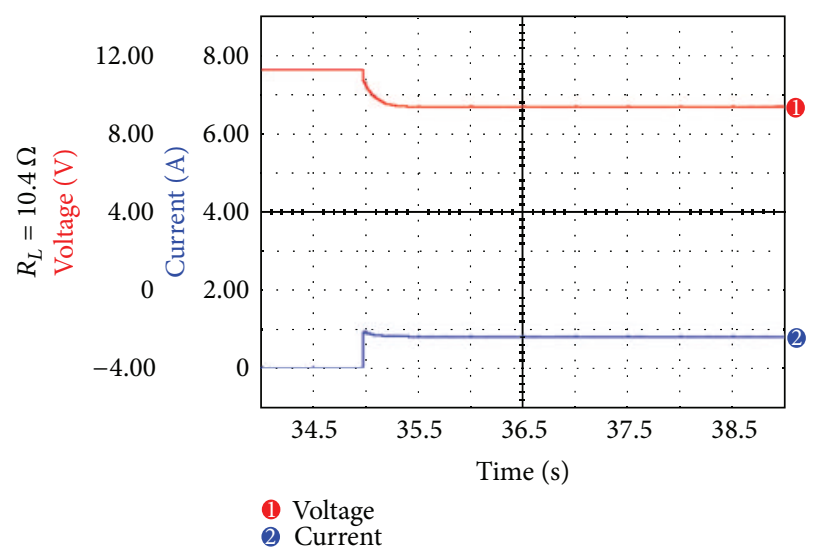

FIGURE 9: The simulated waveform of voltage and current for current loading testing. 
Table 1 demonstrates the promising performance when 120 testing vectors were tested. A good accuracy of the proposed RBF neural network approach was ascertained. The average accuracy rate of 120 testing vectors is $95.5 \%$. Moreover, the average accuracy rates of 14 levels of load current are higher than 94\%, and there is only 1 level of load current that is lower than $94 \%$. The lowest accuracy rate of 15 levels of load current is $93.7 \%$. The effectiveness of the proposed method has been validated in the experiments. The testing results show that the proposed RBF neural network based method can estimate the parameters of PEMFC easily and accurately.

\section{Conclusions}

Based on the hybrid estimation method of RBF neural network and EPSO algorithm, this paper has proposed a method to accurately and reliably estimate the parameters of PEMFC. To demonstrate the effectiveness of the proposed parameters measurement and estimation method, the method has been tested on a practical PEMFC stack. Good accuracy of the proposed hybrid equivalent circuit parameters estimation method was obtained.

\section{Acknowledgments}

The author would like to express his acknowldgement to the National Science Council of ROC for the financial support under Grant no. NSC 100-2221-E-129-001.

\section{References}

[1] M. Yan, L. Fan, and P. Sun, "Constant voltage output in proton exchange membrane fuel cell under fuzzy sliding mode control," Advances in Information Sciences and Service Sciences, vol. 4, no. 20, pp. 180-187, 2012.

[2] J. He and H. Jin, "Research on temperature effect of PEMFC and it's realization of control system," Journal of Convergence Information Technology, vol. 7, no. 16, pp. 97-105, 2012.

[3] K. J. Runtz and M. D. Lyster, "Fuel cell equivalent circuit models for passive mode testing and dynamic mode design," in Proceedings of the 2005 Canadian Conference on Electrical and Computer Engineering, pp. 794-797, Saskatoon, Canada, May 2005.

[4] A. Forrai, H. Funato, Y. Yanagita, and Y. Kato, "Fuel-cell parameter estimation and diagnostics," IEEE Transactions on Energy Conversion, vol. 20, no. 3, pp. 668-675, 2005.

[5] W. Y. Chang, "Application of current switching method to estimate the model parameters of proton exchange membrane fuel cell," Simulation Modelling Practice and Theory, vol. 18, no. 1, pp. 35-50, 2010.

[6] J. Larminie and A. Dicks, Fuel Cell Systems Explained, John Wiley \& Sons, Landon, UK, 2nd edition, 2003.

[7] W. Y. Chang, "Wind energy conversion system power forecasting using radial basis function neural network," in Proceedings of the 2nd International Conference on Engineering and Technology Innovation, Paper No. S1003, KaoHsiung, Taiwan, November 2012.

[8] Y. M. Zhang, Y. B. Zhang, and L. Z. Lin, "Application of genetic algorithm and RBF neural network in network flow prediction," in Proceedings of the 3rd IEEE International Conference on Computer Science and Information Technology (ICCSIT '10), pp. 298-301, Chengdu, China, July 2010.

[9] Y. Zhang, Q. Zhou, C. Sun, S. Lei, Y. Liu, and Y. Song, "RBF neural network and ANFIS-based short-term load forecasting approach in real-time price environment," IEEE Transactions on Power Systems, vol. 23, no. 3, pp. 853-858, 2008.

[10] J. Kennedy and R. Eberhart, "Particle swarm optimization," in Proceedings of the 1995 IEEE International Conference on Neural Networks, vol. 4, pp. 1942-1948, Perth, Australia, December 1995.

[11] X. Qian, M. Cao, Z. Su, and J. Chen, "A hybrid particle swarm optimization (PSO)-simplex algorithm for damage identification of delaminated beams," Mathematical Problems in Engineering, vol. 2012, Article ID 607418, 11 pages, 2012.

[12] C. M. Huang and F. L. Wang, "An RBF network with OLS and EPSO algorithms for real-time power dispatch," IEEE Transactions on Power Systems, vol. 22, no. 1, pp. 96-104, 2007.

[13] R. C. Ebehart, P. K. Simpson, and R. W. Dobbins, Computational Intelligence PC Tool, Academic Press Professional, New York, NY, USA, 1996.

[14] Z. A. Bashir and M. E. El-Hawary, "Applying wavelets to shortterm load forecasting using PSO-based neural networks," IEEE Transactions on Power Systems, vol. 24, no. 1, pp. 20-27, 2009.

[15] W. Y. Chang, "Estimation of the equivalent circuit parameters for proton exchange membrane fuel cell using loading technique," in Proceedings of the 9th Asia-Pacific International Symposium on Combustion and Energy Utilization, pp. 396-400, Beijing, China, November 2008. 


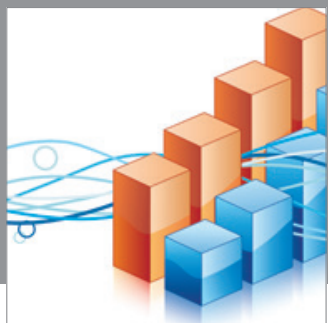

Advances in

Operations Research

mansans

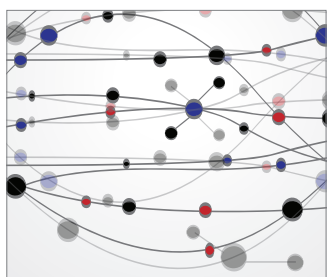

The Scientific World Journal
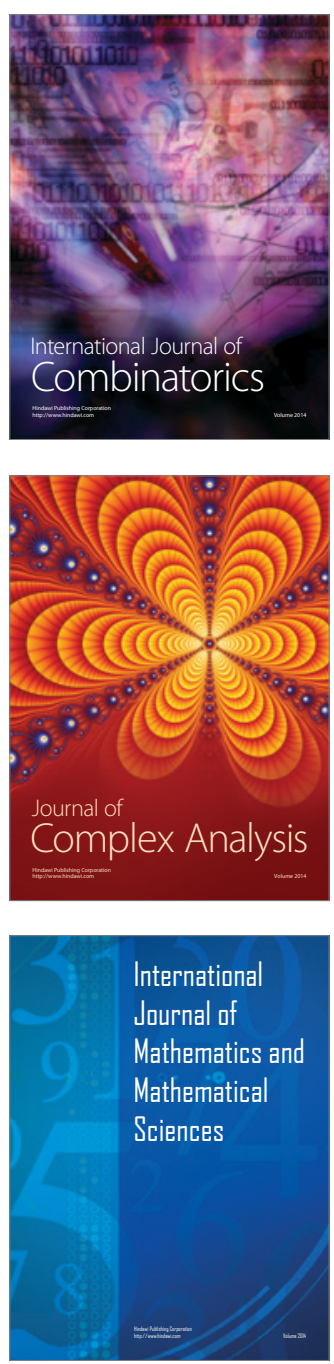
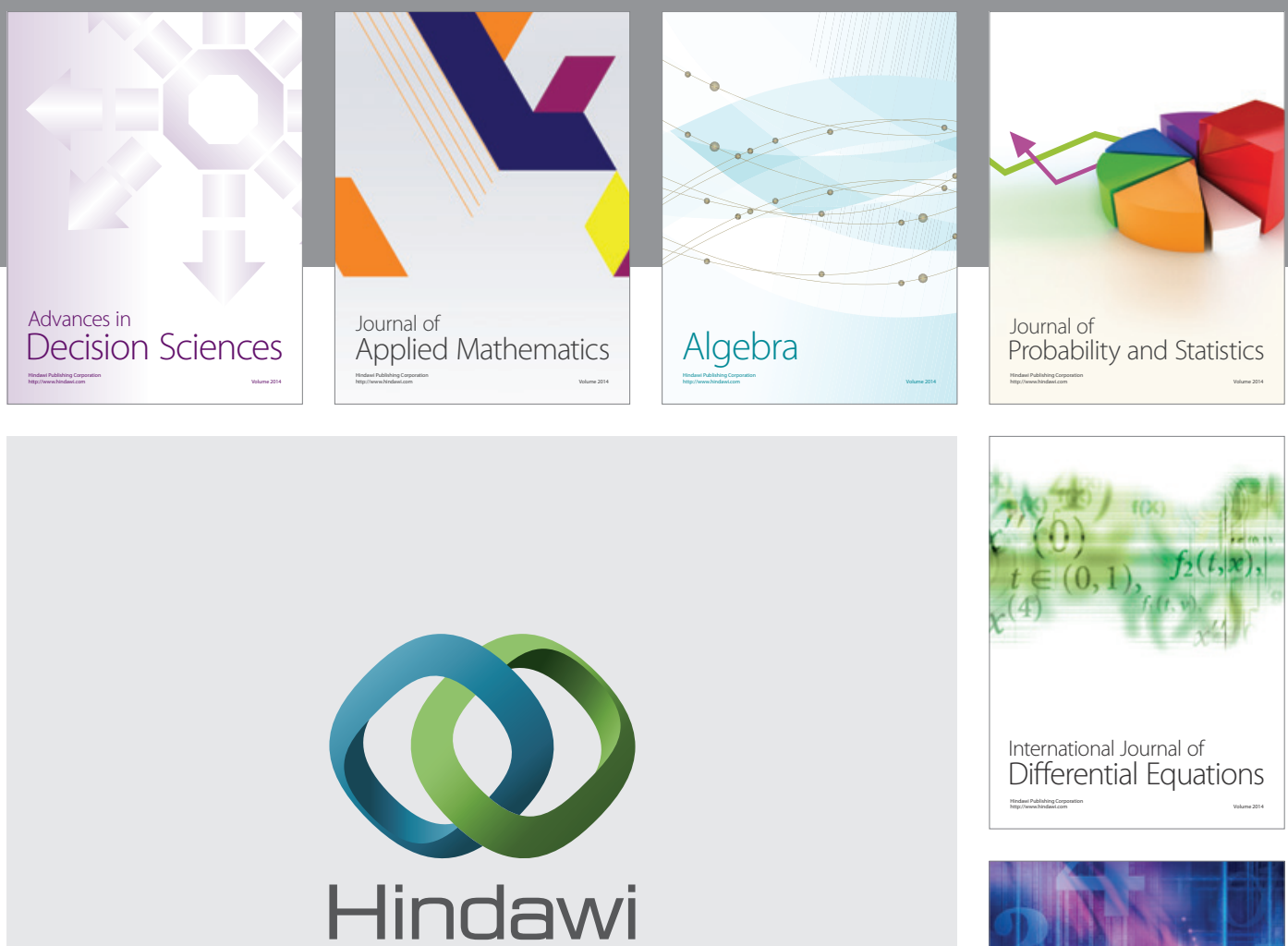

Submit your manuscripts at http://www.hindawi.com
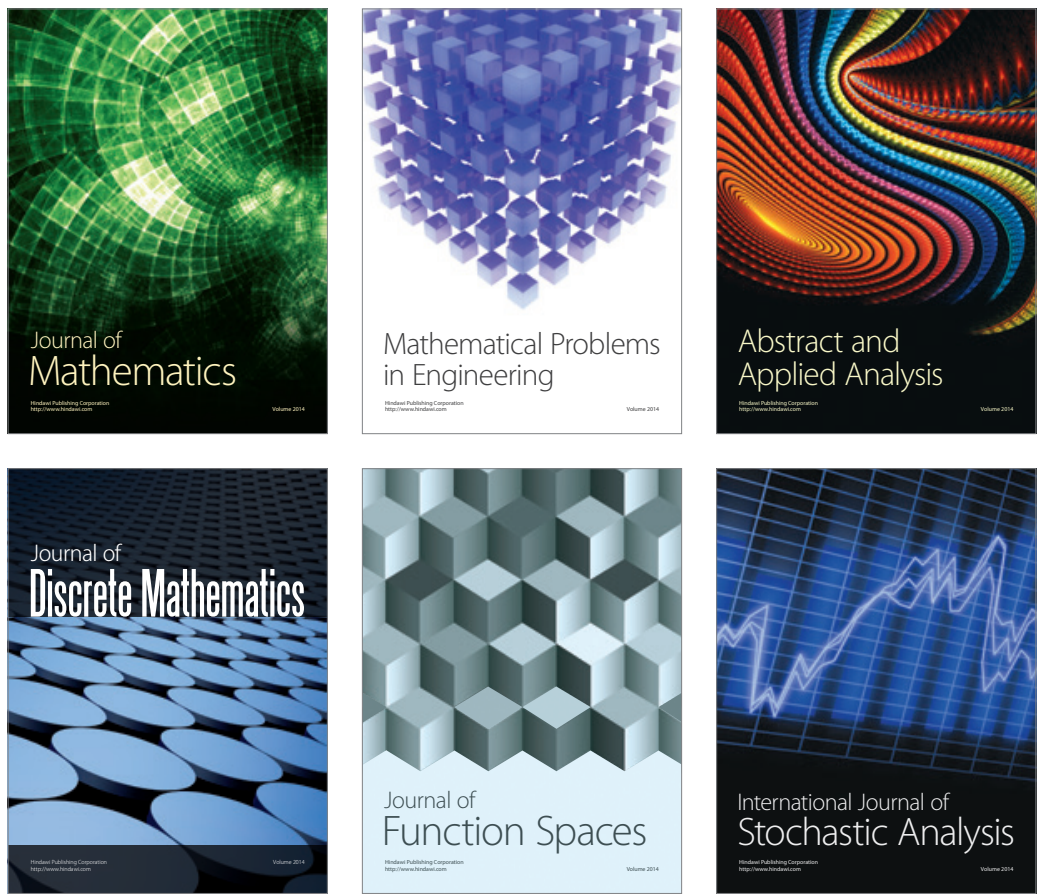

Journal of

Function Spaces

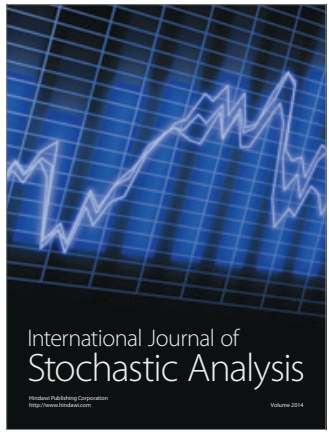

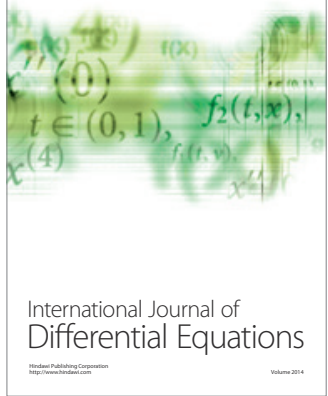
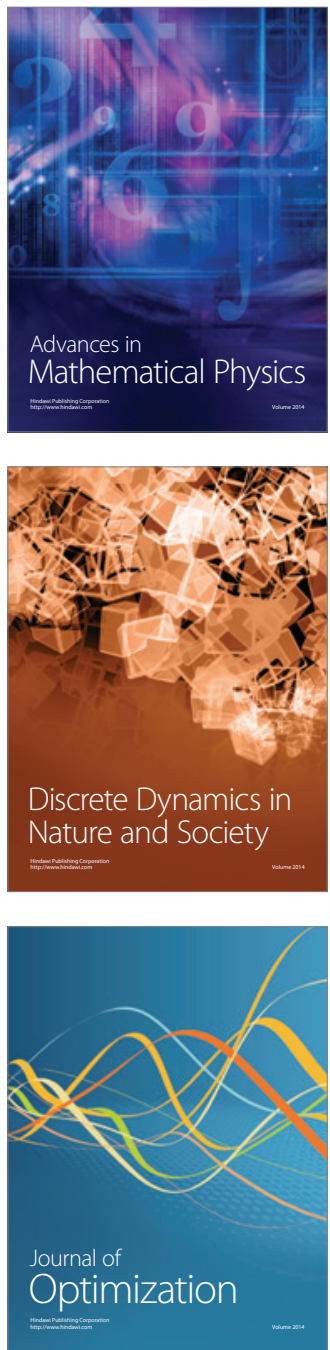